\title{
Reducing hospital acquired infections and professional exposures to blood borne infections through training, surveillance and intervention
}

\author{
Thi Anh Thu Le \\ Cho Ray Hospital, Ho Chi Minh City, Vietnam
}

Int J Infect Contr 2007, 3:1 doi:10.3396/03-01-08-007

Available from: http://www.ijic.info

\begin{abstract}
:
Implementation of infection control measures, including training, surveillance and intervention, at Cho Ray Hospital has been successful in reducing the number of hospital acquired infections and professional exposures to blood borne infections, despite severe overcrowding and a growing number of HIV admissions at the hospital.
\end{abstract}

\section{Introduction}

Hospitals in Vietnam suffer from overcrowding, a lack of resources, and many other challenges. The infrastructure for basic infection control is minimal and the application of universal precautions has been limited in patient care areas and laboratories due to a lack of facilities and knowledge. Infection control in Vietnam remains a developing field, which has only been established since 1997, and the scale of hospital acquired infections in Vietnamese hospitals is largely not quantified.

Cho Ray hospital is a 1705-bed tertiary care referral university centre for Southern Vietnam. The hospital's bed occupancy rate in 2006 was 144\%. There are 8 surgical departments and around 95 operations are performed daily. Approximately 300 emergency patients are admitted and 3000 out-patients are consulted per day.

The Infection Control (IC) team at Cho Ray Hospital has been established since the beginning of 2000 .

\section{Challenges}

- Overcrowding: like all hospitals in Vietnam, Cho Ray Hospital suffers from overcrowding, with a bed occupancy rate of $144 \%$ in 2006.

- Infection control is a relatively new concept.
- The number of HIV admissions is increasing however, prior to July 2001, there were no established protocols for occupational exposures.

\section{Actions}

The IC team at Cho Ray Hospital has conducted effective infection control activities as follows:

Training in infection control:

o Organisation of ongoing training courses about basic infection control for staff at Cho Ray Hospital in addition to staff at other hospitals in Southern Vietnam (Table 1). Informal education is also available continuously on units and in departments.

o Publication of two official infection control manuals ('Infection Control Manual - Practical Guidelines' and 'Guidelines to prevent exposure to HIV, HBV and HCV for Health Care Workers') and a web-based CD.

o Distribution of pamphlets to educate staff about the importance of hand washing and the prevention of exposure to blood borne pathogens. 


\section{Surveillance:}

o Surveillance of the 4 main types of HAI has been conducted, namely nosocomial pneumonia, surgical site infections, nosocomial urinary tract infections and bloodstream infections.

o The IC team observed for non-adherence to established infection prevention and control measures and potentially unsafe practices.

Table 1: Number of staff receiving training about basic infection control

\begin{tabular}{|c|c|c|c|c|}
\hline Year & $\begin{array}{c}\text { Total } \\
\text { participants }\end{array}$ & $\begin{array}{c}\text { Participants } \\
\text { from provincial } \\
\text { hospitals }\end{array}$ & $\begin{array}{c}\text { Participants } \\
\text { from hospitals } \\
\text { in Ho Chi Minh } \\
\text { city }\end{array}$ & $\begin{array}{c}\text { Participants } \\
\text { from Cho Ray } \\
\text { Hospital }\end{array}$ \\
\hline 1999 & 273 & 90 & 81 & 102 \\
\hline 2000 & 238 & 63 & 69 & 106 \\
\hline 2001 & 276 & 96 & 82 & 95 \\
\hline 2002 & 230 & 60 & 84 & 94 \\
\hline 2003 & 478 & 166 & 124 & 188 \\
\hline 2004 & 1048 & 107 & 109 & 832 \\
\hline 2005 & 4139 & 407 & 171 & 3561 \\
\hline 2006 & 4015 & 72 & 180 & 3763 \\
\hline
\end{tabular}

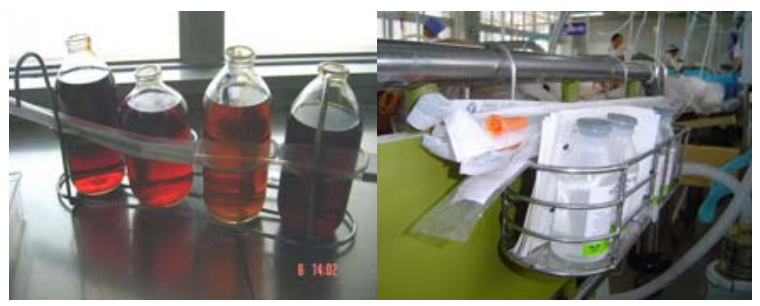

Before intervention

After intervention

Figure 1: Changes to suction procedures

\section{Interventions:}

o The IC team developed and standardised procedures for hospital infection control, such as the schematic presentation of procedures for the disinfection of equipment and linen.

o A number of procedural interventions were made, including:

- $\quad$ improvements to suction procedures (Figure 1);

- $\quad$ the use of alcohol-based hand sanitiser where there is an inadequate number of accessible sinks in patient care areas (Figure 2); and

- $\quad$ application of safe injection procedures (Figure 3).

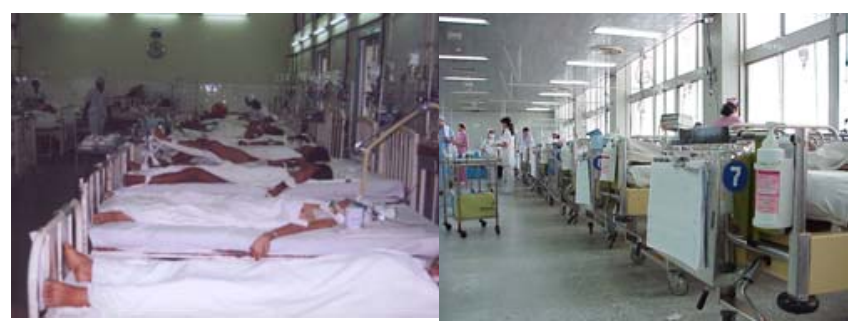

Before intervention

After intervention

Figure 2: Alcohol based sanitiser installed bedside in the overloaded ICU with an inadequate number of sinks

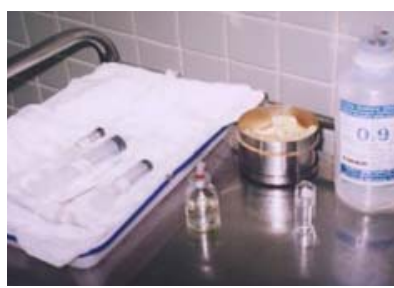

Before intervention

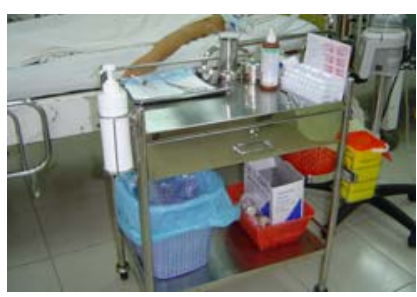

After intervention
Figure 3: Safe injection procedures were established

o Initiation of programmes for the prevention of exposure to blood borne infections for health care workers, including:

- $\quad$ modification of procedures and training of staff to adopt safer work practices;

- $\quad$ provision of sharps disposal containers that meet optimal performance criteria (Figure 4);

provision of sufficient personal protective equipment;

- $\quad$ implementation of a reporting system for occupational exposures; and

- implementation of an HBV vaccination programme.

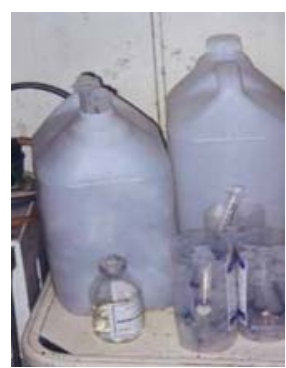

Before intervention

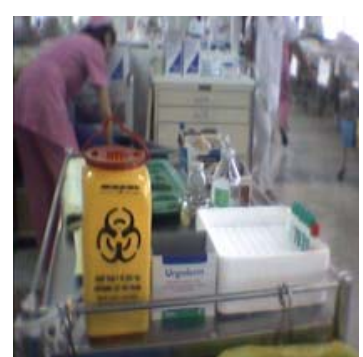

After intervention
Figure 4: Sharps disposal containers were supplied

\section{Results}

The outcomes of these infection control activities are as follows:

- The hospital acquired infection rate decreased gradually between 2001 and 2004, and then significantly since 2004 (Figure 5).

- The establishment of a comprehensive occupational exposures prevention programme for hospital staff (including educational programs, the provision of protective barrier equipment and the provision of needle disposal units) has been successful in preventing HIV-occupational exposures. The numbers of HIVoccupational exposures was reduced significantly, 


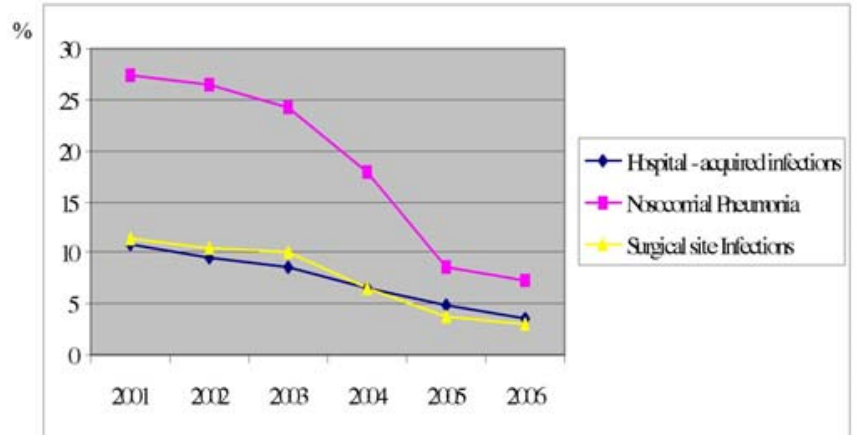

Figure 5: Hospital acquired infection rates at Cho Ray Hospital, by year

from 1.3 cases /month in the first 6 months of 2001 to 0.08 cases/month in 2002, and remained low at 0.25 cases/month in 2006 despite the increasing number of HIV-infected patients admitted to Cho Ray Hospital $(\mathrm{P}=0.007)$ (Figure 6).

- In addition, the programme (which supplied health care workers with a better understanding of HIV and provided further psychological support in private consultation) helped to reduce psychological stress following HIV exposures.

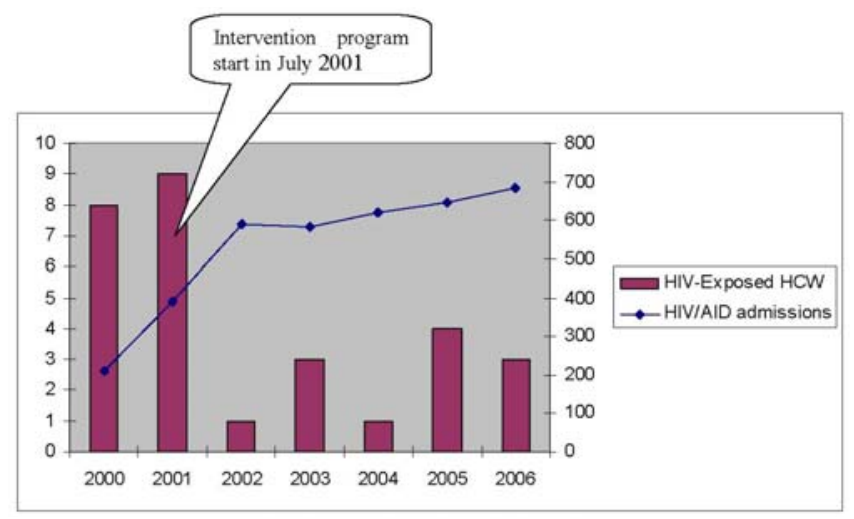

Figure 6: Number of HIV-occupational exposures at Cho Ray hospital, by year-number of individuals

\section{Conclusion}

Despite a lack of awareness about infection control (prior to the establishment of an infection control team in 2000), severe overcrowding and limited resources, the infection prevention and control measures implemented by the IC team, namely training, surveillance and intervention, have been successful in reducing the number of hospital acquired infections experienced at Cho Ray Hospital.

In addition to protecting patients from hospital acquired infections, the introduction of a comprehensive occupational exposures prevention programme has helped to protect health care workers at the hospital against blood borne infections and has significantly reduced the number of HIV-occupational exposures at the hospital.

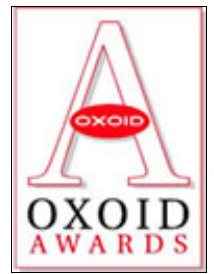

This publication has been adapted from the original submission by the authors for the Oxoid Infection Control Team of the Year Awards 2006/2007 (www.oxoid.com) and is being reproduced with permission of Oxoid Ltd. 